\title{
L’inclusion comme théorie pratique
}

\author{
Inclusion as practice-theory
}

Inclusão como teoria prática

\author{
Éric Plaisance \\ Professeur émérite, Université Paris Descartes Université de Paris, Paris, France. \\ erplaisance@gmail.com \\ https://orcid.org/0000-0002-7047-4194
}

Recebido em 23 de setembro 2020

Aprovado em 24 de setembro de 2020

Publicado em 10 de novembro de 2020

\section{RÉSUMÉ}

En reprenant l'expression "théorie pratique" qu'Émile Durkheim a utilisée pour désigner la pédagogie, la notion d'inclusion est analysée de manière critique en examinant son caractère normatif selon les aspects suivants: son étymologie latine, ses usages initiaux dans les politiques internationales d'éducation et leurs évolutions vers la valorisation de la diversité; ses variations de signification selon les pays en fonction de leurs politiques et de leurs institutions, en prenant l'exemple de l'Angleterre, de l'Italie, du Brésil; sa signification par rapport à deux notions : l'exclusion à laquelle elle s'oppose; l'intégration dont elle se démarque; ses conséquences paradoxales selon les analyses des sciences humaines et sociales contemporaines, avec et au-delà de Durkheim. Enfin, pour dépasser les contradictions de la notion d'inclusion, l'accessibilité peut être mobilisée comme concept unificateur et opérationnel et comme nouvel idéal normatif.

Mots-clés: Inclusion; Éducation inclusive; Accessibilité.

\section{ABSTRACT}

By using the expression "practical theory" that Émile Durkheim has used to designate pedagogy, the notion of inclusion is analyzed critically by examining its normative character according to the following aspects: its Latin etymology, its initial uses in politics international education programs and their evolutions towards valuing diversity; its variations in meaning between countries depending on their policies and institutions, taking the example of England, Italy, Brazil; its meaning in relation to two notions: the exclusion of with it opposes; the integration from which it stands out; its paradoxical consequences according to analyzes of contemporary human and social sciences, with and beyond Durkheim; accessibility perspectives that embody inclusion. Finally, to overcome the contradictions of the notion of inclusion, accessibility can be mobilized as a unifying and operational concept and as a new normative ideal.

Keywords: Inclusion; Inclusive education; Accessibility. 
http://dx.doi.org/10.5902/1984686X55337

\section{RESUMO}

Ao utilizar a expressão "prática", que Émile Durkheim adotou para projetar a pedagogia, a noção de inclusão é analisada criticamente, examinando seu caráter normativo de acordo com os seguintes aspectos: sua etimologia latina, seus usos iniciais nas políticas internacionais de educação e seu desenvolvimento rumo à valorização da diversidade; suas variações de significação conforme os países em função de suas políticas e suas instituições, tendo como exemplo a Inglaterra, a Itália e o Brasil; seus sentidos em relação às duas noções: a exclusão àquilo que se opõe; a integração demarcada; suas consequências paradoxais segundo as análises das ciências humanas e sociais contemporâneas, com e para além de Durkheim; as perspectivas de acessibilidade que concretizam a inclusão. Por fim, para superar as contradições da noção de inclusão, a acessibilidade pode ser mobilizada como um conceito unificador e operacional e como um novo ideal normativo.

Palavras-chave: Inclusão; Educação inclusiva; Acessibilidade.

\section{Introduction}

Émile Durkheim a utilisé l'expression théorie pratique pour désigner la pédagogie, qui ne vise pas, selon lui, à connaitre scientifiquement les faits éducatifs mais à orienter la pratique des professeurs. J'utilise l'expression en l'élargissant au-delà de la pédagogie pour en faire un instrument d'analyse critique de l'ambition sociale revendiquée par la notion d'inclusion, c'est à dire pour ne pas la prendre spontanément au premier niveau d'adhésion mais dans une perspective de vigilance épistémologique, en adhérant à l'orientation critique de Gaston Bachelard.

Il convient alors de considérer la notion dans sa présence dans les politiques éducatives, sur le plan international et national, en montrant son caractère de prescription pour modifier ou améliorer les politiques et les pratiques. On peut ainsi être en mesure de montrer les élargissements des significations prescriptives au cours du temps, par exemple à l'UNESCO qui insiste de plus en plus sur la prise en compte de la diversité des enfants plus que des besoins dits spéciaux, mais on relève aussi les grandes variations des interprétations selon les pays concernés.

La question fréquemment soulevée par les chercheurs et les praticiens est celle des équivoques et des contradictions dans les mises en pratique. Quelques exemples sont ultérieurement fournis, parmi lesquels des enquêtes de type ethnographique dans des établissements scolaires en France.

Enfin, on peut se demander s'il est judicieux de dépasser la notion d'éducation inclusive vers le nouvel idéal normatif d'accessibilité de l'environnement pour son ouverture à l'autre et à sa participation sociale. 


\section{Rappel historique: l'éducation et la pédagogie pour Émile Durkheim}

Durkheim distingue soigneusement les deux termes. L'éducation relève des faits sociaux et, selon sa célèbre définition, elle est "l'action exercée sur les enfants par les parents et les maitres" (DURKHEIM, 1980, p. 69). De son coté, a pédagogie "consiste non en actions mais en théories". Et l'auteur ajoute: "Ces théories sont des manières de concevoir l'éducation, non des manières de pratiquer."

S'ensuit une discussion d'abord sur la science de l'éducation (expression utilisée au singulier par Durkheim), ensuite sur la conception de la pédagogie qui se réduirait à art. Durkheim défend la possibilité d'une approche scientifique de l'éducation car celle-ci consiste en objets identifiables, elle est en mesure de produire des connaissances sur les causes de phénomènes, d'en déterminer leurs effets, plus exactement en étant attentif à la genèse et au fonctionnement des systèmes éducatifs. Cependant,

les théories que l'on appelle pédagogiques sont d'une tout autre sorte [...] Leur objectif n'est pas de décrire ou d'expliquer ce qui est ou ce qui a été, mais de déterminer ce qui doit être [...] Elles ne se proposent pas d'exprimer fidèlement des réalités données mais d'édicter des préceptes de conduite (DURKHEIM, 1980, p. 77).

La pédagogie est-elle alors un art, c'est-à-dire une pratique pure, par exemple se réduit-elle au fait d'un éducateur habile praticien qui a une expérience personnelle. Elle occupe en réalité une position intermédiaire entre la pratique dite pure et le projet scientifique, elle relève d'une "attitude mentale intermédiaire" (ibid, p.79).

De même que pour les théories médicales, politiques ou stratégiques, la pédagogie comporte une dimension théorique mais prioritairement pour diriger les pratiques. II s'agit donc d'exprimer le caractère mixte de ces spéculations en les dénommant des théories pratiques. La conclusion de cette analyse est donc de définir la pédagogie comme une théorie pratique de ce genre. "Elle n'étudie pas scientifiquement les systèmes d'éducation mais elle y réfléchit en vue de fournir à l'activité de l'éducateur des idées qui le dirige."

Notre hypothèse de travail, à partir de ce rappel de la définition de la pédagogie par Durkheim, est d'utiliser la notion de théorie pratique comme instrument d'analyse des notions actuellement diffusées internationalement principalement dans le champ éducatif selon une perspective normative : inclusion sociale, inclusion scolaire, éducation inclusive. 
http://dx.doi.org/10.5902/1984686X55337

\section{L'inclusion: étymologie et usages}

Les origines latines du mot inclusion nous confrontent d'emblée à une situation paradoxale. En latin, clausus est le participe passé du verbe claudere et signifie clos. Dans le même sens, claustra c'est la fermeture, le lieu clos, l'enclos, voire la prison. En français, les mots cloître et claustration proviennent de la même origine. On est donc face à l'idée de fermeture et de clôture.

Doit-on alors considérer que l'inclusion, dans le sens actuellement valorisé, signifie l'isolement du lieu fermé sur lui-même ou encore la ségrégation des sujets à l'intérieur de ce lieu (PELLETIER, 2020). Comme le souligne le spécialiste brésilien du droit à l'éducation, Carlos Roberto Jamil Cury, in-clure serait "faire entrer dans la clôture du cloître" (CURY, 2009, p. 41). Le même auteur ajoute ensuite que l'inclusion et l'exclusion entretiennent des relations dialectiques, que l'une n'existe pas sans l'autre, et que, par conséquent, une réflexion qui s'appuie sur la notion d'éducation inclusive ne peut ignorer les thèmes de l'inégalité et de la discrimination, précisément pour les contrecarrer.

Sa définition de l'éducation inclusive, très proche de celles de l'UNESCO, est finalement bien opposée à l'idée de fermeture et valorise la référence au droit à la scolarisation pour tous:

Dans son principe, l'éducation inclusive correspond à une modalité de scolarisation où ceux qui étudient ont les mêmes droits que leurs pairs, sans discrimination de sexe, de race, d'ethnie, de religion et de capacité. Ils disposent donc du droit de fréquenter les mêmes établissements et de participer aux activités de élèves de leur âge. (CURY, 2009, p. 42)

La notion d'inclusion dans le sens précédent est d'un usage récent dans la langue française. Précédemment, le vocabulaire de l'inclusion s'appliquait seulement aux choses et non aux personnes, par exemple l'inclusion d'une clause juridique dans un texte de droit, l'inclusion d'une pièce de métal dans une autre. Or, à partir des années 1990 au niveau européen, puis dans les années 2000 au niveau français, le terme a été progressivement utilisé dans les milieux scientifiques et professionnels impliqués dans les revendications des politiques éducatives, pour s'appliquer aux situations scolaires, aux enfants et aux groupes d'enfants.

Dans les années qui ont précédé la préparation de la loi française de 2005 sur les personnes handicapées, le Conseil National Handicap: sensibiliser, informer, former ${ }^{1}$ avait cherché à susciter une véritable mutation culturelle visant à "désinsulariser" le handicap à tous niveaux. II a donc soutenu le vocabulaire de l'inclusion et de l'éducation inclusive, une 
http://dx.doi.org/10.5902/1984686X55337

position clairement exprimée par Julia Kristeva dans sa lettre ouverte au Président Chirac, en 2003, dont le titre était calqué sur le livre de Diderot sur les aveugles:

\begin{abstract}
Je comprends ceux qui préfèrent remplacer le terme d' intégration par celui d'insertion ou d'inclusion. Loin d'être politically correct, ce souci sémantique invite non pas à 'intégrer' au sens d'effacer la différence mais à inclure des personnes différentes dans l'espace public (écoles, entreprises, etc.), à condition d'y aménager des parcours individualisés et des accompagnements singuliers (KRISTEVA, 2003, p. 51).
\end{abstract}

Certains Instituts Universitaires de Formation des Maitres - IUFM se sont alors fait les relais de ces orientations soutenues par l'innovation de vocabulaire pour promouvoir un autre regard sur la présence des enfants en situation de handicap dans le milieu scolaire ordinaire. Pourtant, ni la loi de janvier 2002 de rénovation de l'action sociale et médicosociale ni la loi de 2005 "sur l'égalité des droits et des chances, la participation et la citoyenneté des personnes handicapées", qui rénovait celle de 1975, ne s'étaient emparées de ces innovations pour appuyer les politiques. C'est seulement la loi de 2013 sur la refondation de l'École de la République qui fait utilise la notion d'inclusion scolaire dans les termes suivants : le service public d'éducation "veille à l'inclusion scolaire de tous les enfants, sans aucune distinction" (FRANCE, 2013, Art. $2^{\circ}$ ).

L'inclusion se décline le plus souvent sous forme d'adjectif. On repère, sous la forme la plus générale, les politiques dites "inclusives" dans une société qui devrait être "inclusive" et, de manière plus spécifique, l'éducation "inclusive", l'entreprise "inclusive", etc. On est donc en mesure de constater une inflation des usages en tous domaines, ce qui contribue sans doute aux confusions de compréhension des significations. II n'est pas étonnant que ces usages soient l'objet de débats, voire de controverses, tant sur le plan des définitions que sur celui des conséquences qu'ils impliquent, en termes d'institutions, de professionnels et de pratiques (TOULLEC-THERY, 2020).

C'est précisément pour éviter les ambiguïtés du terme inclusion que Charles Gardou préfère l'adjectif inclusif, en opposition à exclusif. II repère dans l'inclusion, des dimensions qui renvoient à l'idée l'occlusion, de clôture, voire de réclusion (GARDOU, 2012). L'adjectif lui semble plus adéquat et pertinent, car il témoigne d'un double refus: celui d'un monde dont certains se prétendraient les propriétaires exclusifs; celui de la mise à l'écart "de ceux que l'on juge gênants, étrangers, incompatibles." Dès lors, Gardou défend la perspective d'une société inclusive qui a pour visée de "réunifier les univers sociaux hiérarchisés pour forger un nous." II revendique une révolution dans les manières de penser, pour susciter de nouvelles Lumières, pour nous défaire des préjugés et des obscurantismes en nous libérant 
http://dx.doi.org/10.5902/1984686X55337

de nos bastilles intérieures. Ces nouvelles Lumières devraient permettre de dessiner la matrice d'un univers social rassemblé, qui reconnait la vulnérabilité comme condition commune et fait place à ses diverses expressions comme aux droits qui en émanent (GARDOU, 2012; 2014).

La revendication formulée ainsi d'un futur sociétal renouvelé témoigne d'une orientation de pensée explicitement utopique, comme une nécessité éthique devant contrecarrer les discriminations (PLAISANCE, 2009; 2010). La visée inclusive se présente alors comme un devoir être, sous un aspect normatif, clairement assumé par l'auteur.

\section{Les mouvements internationaux et leurs évolutions}

Sur le plan international c'est l'expression éducation inclusive, qui est diffusée largement, plus que celle d'inclusion scolaire, utilisée dans la législation française de 2013, sans doute du fait de l'usage mondialement généralisé de la langue anglaise ainsi que des particularités du système éducatif français.

Rappelons que le point de départ du mouvement international est habituellement fixé au moment de la rencontre de Salamanque (Espagne) en 1994, organisée par l'UNESCO dans le prolongement de la décennie des personnes handicapées de l'ONU et réunissant les représentants de 92 pays. La déclaration et le cadre d'action adoptés enjoignaient les gouvernements de dépasser l'éducation traditionnelle spéciale et de s'engager vers une éducation pour tous et en particulier pour des enfants à besoins éducatifs spéciaux, définis de la manière suivante: "tous les enfants et adolescents dont les besoins découlent de handicaps ou de difficultés d'apprentissage" (UNESCO, 1994, p. 1). Ce sont alors les écoles dites intégratrices qui devaient partir du "principe fondamental que tous les élèves d'une communauté doivent apprendre ensemble, dans la mesure du possible, quels que soient leurs handicaps et leurs difficultés." De plus, de telles écoles seraient "le moyen le plus efficace de combattre les attitudes discriminatoires, en créant des communautés accueillantes."

Les énoncés de cette déclaration ne sont pas encore bien stabilisés à cette époque. Le vocabulaire de la traduction française est celui de l'intégration et n'est pas encore celui de l'inclusion', et les enfants concernés sont souvent référés au handicap ou au besoin dit spécial. II est significatif que la formation initiale des maîtres, très rapidement évoquée, renvoie à une approche positive du handicap et à des dispositifs d'appui, même si, par ailleurs, il est bien question de l'éducation pour tous. 
http://dx.doi.org/10.5902/1984686X55337

Les documents plus récents de I'UNESCO adoptent un vocabulaire bien différent. La notion d'inclusion est au premier plan et le public scolaire visé est caractérisé par sa diversité et ne se limite ni aux élèves en situation de handicap ni à ceux qui ont des besoins spéciaux. Ainsi, les "principes directeurs pour l'inclusion dans l'éducation" font référence à l'ensemble des apprenants et non à un type spécifique d'élèves:

L'éducation inclusive renforce la capacité du système éducatif à atteindre tous les apprenants et peut donc être comprise comme une stratégie pour réaliser l'éducation pour tous (EPT) [...] L'éducation est ainsi envisagée comme un processus qui vise à prendre en compte et à satisfaire la diversité des besoins de tous (UNESCO, 2009, p.8-9).

Dans le même sens, une série de guides pratiques de l'UNESCO sur la promotion de la nouvelle figure de l'enseignant dans un contexte inclusif énonce clairement qu'il faut dépasser l'éducation des besoins spéciaux vers un paradigme plus large de l'éducation inclusive, car il s'agit d'adopter une vision holistique de l'éducation pour tous les apprenants (UNESCO, 2013).

La déclaration UNESCO de 2015 à Incheon (République de Corée) fait encore un pas de plus en articulant inclusion, équité et qualité en faveur des apprentissages, et en adoptant la formulation suivante : "Assurer une éducation inclusive et équitable de qualité et promouvoir des possibilités d'apprentissage tout au long de la vie pour tous" (UNESCO, 2015).

Enfin, en 2020, l'Unesco a publié un rapport détaillé sur l'éducation et l'inclusion qui s'appuie sur des données précises recueillies dans les pays du monde entier et les présente de manière le plus souvent sous forme de tableaux de données statistiques. Ce bilan international renforce encore le point essentiel selon lequel l'inclusion en éducation concerne des pratiques qui dépassent les seuls enfants avec besoins spéciaux mais englobent la diversité des apprenants en construisant un sens de l'appartenance pour tous (UNESCO, 2020). En ce sens, la diversité, au lieu d'être un problème, serait une occasion favorable aux changements des pratiques éducatives. Cependant, le rapport mentionne que, parmi les pays enquêtés, 68\% fournissent bien une définition de l'éducation inclusive, mais $57 \%$ ne se réfèrent pas aux groupes sociaux marginalisés et $26 \%$ réduisent l'éducation inclusive aux handicaps et aux besoins spéciaux.

En conformité avec ces orientations, l'Agence européenne pour la promotion des besoins spéciaux, ainsi dénommée encore dans le début des années 2000, a modifié son appellation pour devenir l'Agence européenne pour l'éducation adaptée et inclusive. Mais 
http://dx.doi.org/10.5902/1984686X55337

dans son bilan de littérature internationale de 2013, l'Agence se référait déjà à l'idée de l'inclusion comme processus de changement ayant pour but de répondre à la diversité en identifiant et en combattant les phénomènes de discrimination (EUROPEAN AGENCY, 2013, p. 11-17).

Dans cette évolution, c'est sans doute la question du droit qui a été déterminante, c'est-à-dire le droit de tous à accéder à l'école commune et, ensuite, d'y bénéficier d'un enseignement de qualité, y compris, pour certains élèves, de dispositifs d'accompagnement (PRUDHOMME, 2016; TREMBLAY 2012).

\section{Les variations nationales}

Pour ne pas utiliser le terme d'inclusion comme un mot valise, un simple slogan, une notion-parapluie (selon l'expression en portugais), et exercer sa vigilance critique, il est pertinent d'examiner les variations selon les pays pour relativiser l'aspect trompeusement homogénéisant du terme (BENOIT; PLAISANCE, 2009; RAHME, 2013).

L'éducation inclusive est ancrée dans des configurations spécifiques et "ce qui caractérise et différencie les approches nationales entre elles, ce sont d'abord les conceptions, représentations et rationalités autour desquelles s'organisent les systèmes scolaires" (EBERSOLD; PLAISANCE; ZANDER, 2016, p. 28).

Certains pays adoptent une approche essentialiste des besoins éducatifs particuliers, en mettant prioritairement l'accent sur les difficultés de l'élève et en le situant dans un écart par rapport à une norme scolaire. On met alors en place des mesures de soutien spécifiques, y compris par des classes spéciales, par exemple pour des élèves considérés handicapés ${ }^{3}$.

D'autres pays adoptent une approche universaliste qui considère avant tout les capacités des systèmes scolaires à être accessibles à tous et offrent par conséquent une vision plus large des publics scolaires. L'enjeu principal, selon les mêmes auteurs, est alors de penser le besoin éducatif non pas à partir de l'élève mais à partir de l'environnement et de l'interaction éducative qui entoure l'enfant. Dans ce cas, les systèmes éducatifs sont considérés des milieux de vie où les objectifs éducatifs ne se limitent pas aux apprentissages scolaires mais visent une éducation totale de l'enfant ${ }^{4}$. De manière générale, c'est l'ouverture des systèmes éducatifs à la différence, par-delà la création de dispositifs ou la mobilisation de techniques ou de procédures, provient des ambitions du système scolaire en matière d'accès, de réussite et d'insertion sociale. 
http://dx.doi.org/10.5902/1984686X55337

En Angleterre, des propos critiques, voire polémiques, ont été lancés par certains auteurs contre ceux qui ont nommés de manière péjorative, les inclusionnistes, principalement pour réclamer le dépassement des propos compassionnels et la recherche d'indicateurs précis sur les réalités de l'inclusion dans des contextes locaux. Ainsi, Mary Warnock, l'ancienne responsable du célèbre rapport britannique de 1978 sur les besoins éducatifs spéciaux, a remis vivement en cause les perspectives inclusives, en considérant que l'essentiel n'était pas de savoir si les enfants devaient tous être sous le même toit mais s'ils participaient effectivement aux apprentissages (WARNOCK; NORWICH; TERZI, 2010).

En Suède, où une politique volontariste de normalisation et de désinstitutionalisation est entrée en vigueur dès les années 1960, des auteurs sont bien plus nuancés et cherchent à repérer les dilemmes de l'éducation inclusive, en distinguent plusieurs niveaux d'analyse depuis le cadre sociétal le plus global jusqu'au niveau des pratiques (MICHAILAKIS; REICH, 2010).

Face à la nouveauté des perspectives inclusives, l'Italie fait figure d'exception car elle mène une politique radicale depuis les années 70 . La politique éducative pour les enfants handicapés a pris une nouvelle tournure radicale dès la loi de 1977, au nom de l'intégration scolaire dans les classes ordinaires. La loi, qui était une loi d'organisation pédagogique et non une loi portant sur les personnes handicapées, a supprimé les classes différenciées et a donc généralisé la présence des enfants en situation de handicap non seulement dans les écoles ordinaires mais aussi et surtout dans les classes ordinaires. En même temps, cette loi a introduit la programmation didactique et une nouvelle façon d'évaluer le parcours scolaire. Cette option en faveur de l'integrazione scolastica, selon l'expression encore utilisée en Italie, est issue des mouvements sociaux des années soixante, qui l'ont préparée par une critique des asiles d'aliénés, grâce au rôle des associations revendicatives, à une volonté de réforme du fonctionnement de l'école et au rejet de toute situation de marginalisation. Les choix politiques se sont traduits par des mesures concrètes, en particulier par la mise en place d'enseignants, d'abord dénommés spécialistes pour les activités intégratives, puis professeurs de soutien, chargés d'apporter leur appui au professeur de la classe ordinaire en travaillant directement avec lui (DE ANNA; PLAISANCE, 2014). Quarante ans après cette révolution pédagogique, l'intégration à l'italienne, qui est bien une éducation inclusive avant la lettre, reste encore, aux yeux des spécialistes de ce pays, un processus à consolider constamment. L'usage actuel de la notion d'éducation inclusive en Italie ne vise pas à supprimer les acquis de l'intégration 
http://dx.doi.org/10.5902/1984686X55337

scolaire mais plutôt à la compléter en développant de nouvelles collaborations, par exemple des projets qui impliquent l'environnement local et l'adhésion de tous à l'accueil de la diversité ${ }^{\text {. }}$

Quant à la situation au Brésil, elle se révèle dans toute sa complexité, voire ses contradictions entre l'éduction spéciale et l'éducation inclusive. Pourtant, le Brésil est le seul pays qui a posé la notion d'inclusion comme une orientation nationale générale susceptible de guider des actions en tous domaines : pour l'emploi des afro-américains, combinée à une politique de non-discrimination, pour la facilitation d'accès aux transports publics des populations pauvres, pour la lutte contre l'abandon scolaire, pour l'accès à l'école ordinaire des enfants en situation de handicap etc. Dans ces conditions, la notion d'intégration est considérée comme reflétant des conceptions dépassées. C'est l'inclusion qui devient le maitre mot comme application du droit à la diversité. L'éducation inclusive est alors une option politique fondamentale dans le champ éducatif pour une école de qualité pour tous. Ce qui impliquerait des changements importants dans les procédures de gestion de la scolarité, dans la formation des professeurs, dans les méthodologies éducatives et les pratiques collaboratives.

Néanmoins, cette claire affirmation politique en faveur de l'éducation inclusive est paradoxalement combinée avec le maintien de l'éducation spéciale. A un premier niveau, subsistent des institutions publiques spécialisées (pour déficiences visuelles et auditives) et des institutions privées d'accueil d'enfants en situation de handicap, gérées par des associations puissantes, comme des associations de parents, l'association Pestalozzi etc. A un second niveau, les politiques scolaires publiques articulent les deux notions de spécial et d'inclusif, selon la Política Nacional de Educação Especial na Perspectiva da Educação Inclusiva (BRASIL, 2008).

L'éducation inclusive relève de la responsabilité de l'école ordinaire et de ses maitres et, de son côté, l'éducation spéciale se réfère à des services tels que les services d'accueil spécialisé, les salles de ressources, les professeurs spécialisés. Ce serait donc une articulation complexe entre l'enseignement commun inclusif et l'éducation dite spéciale, principalement à travers l'accueil éducatif spécialisé (VOLTOLINI, 2014; 2019; SILVA, 2016; NEVES RODRIGUES et al., 2019; ULLRICH 2019). La question plus radicale est de savoir s'il s'agit de deux faces compatibles dans un projet politique, d'un mariage de raison par souci de conciliation pragmatique, ou d'un concubinage fondamentalement illégitime. 
http://dx.doi.org/10.5902/1984686X55337

En réalité, une redéfinition profonde du rôle de l'éducateur spécialisé serait alors nécessaire: comme une "personne ressource" en lien avec l'ensemble des partenaires professionnels de l'école mais aussi avec les familles, comme une personne pouvant développer des expertises multiples et coopératives au service de l'éducation inclusive. Cette redéfinition dépasserait les anciens clivages entre le spécial et le normal qui ont marqué l'histoire des institutions et des cultures professionnelles (CARVALHO, 2008).

\section{L'inclusion face à l'exclusion}

A l'évidence, la notion la plus spontanément opposée à l'inclusion est celle d'exclusion (RAVAUD; STIKER, 2000a; 2000b). Cette dernière a été consacrée en France dans le domaine des politiques sociales, grosso modo dans les années 90 , en lien avec la question de la nouvelle pauvreté et de la précarité (PAUGAM, 1996). Pourtant, exclusion et inclusion, contrairement aux apparences, ne sont pas des notions symétriques. Alors que la notion d'exclusion est descriptive puisqu'on peut observer des situations où des personnes seraient dites exclues, par exemple de services publics, l'inclusion se réfère à un idéal de fonctionnement éducatif, voire à un idéal social, et la notion est donc normative. La notion d'exclusion a été vivement critiquée par Robert Castel qui la considère comme une notion écran, une notion piège (CASTEL, 2009). Pour lui, l'indigence théorique de la notion vient essentiellement du fait qu'elle "recouvre des situations tellement hétérogènes qu'elle ne permet d'en analyser aucune." . Spécialiste des politiques sociales et de l'évolution du salariat, Robert Castel (2009) met en garde contre l'usage abusif et trompeur de la notion d'exclusion, car elle laisse croire que l'on a seulement à faire à des états, à des positions limites, alors que les situations observées révèlent des processus de désaffiliation. "Dans la plupart des cas, 'l'exclu' est en fait un désaffilié dont la trajectoire est faite d'une série de décrochages par rapport à des états d'équilibre antérieurs plus ou moins stables, ou instables" (CASTEL, 2009, p. 343). Le grand avantage de la conceptualisation en termes d'affiliation et de désaffiliation est de tracer des passages et des continuités entre les situations, au lieu d'oppositions radicales. Ce sont des trajectoires différentes qui caractérisent les individus.

Une autre clarification conceptuelle est présente dans les analyses de Pierre Bourdieu sur les inégalités de scolarisation au sein du même système scolaire. Avec Patrick Champagne, il analyse les évolutions du système d'enseignement en France depuis les années 50, et dénonce les apparences d'une démocratisation qui voit la présence 
http://dx.doi.org/10.5902/1984686X55337

grandissante d'adolescents de catégories populaires au sein de l'enseignement du second degré mais maintient en même temps les mécanismes de reproduction sociale. L'institution scolaire "est habitée durablement par des exclus en puissance" (BOURDIEU; CHAMPAGNE, 1992, p.72). L'école « exclut désormais de manière continue, à tous les niveaux du cursus » et les auteurs ajoutent qu'elle garde ceux qu'elle exclut, et qu'elle se contente de les reléguer dans des filières plus ou moins dévalorisées. Ce sont, selon leur belle expression, des "exclus de l'intérieur" qui "traînent sans conviction une scolarité qu'ils savent sans avenir."

On peut s'inspirer de cette analyse dénonciatrice pour développer un regard critique sur la notion d'inclusion scolaire. Le placement à l'intérieur d'un même lieu ne signifie pas nécessairement la fin des situations de ségrégation. Des enfants peuvent se situer à l'intérieur d'une école ordinaire ou d'une classe ordinaire et, en même temps, faire l'objet de rejets subtils, de marginalisations, par exemple, ne pas participer aux activités, ne pas être dans des processus d'affiliation, selon le vocabulaire de Castel. Les enfants en situation de handicap peuvent être aussi des exclus de l'intérieur. De nombreuses observations ou des enquêtes de terrain montrent ces paradoxes de l'inclusion qui, dans certains cas, peut se retourner en son contraire, se transformer en inclusion excluante, reléguer des enfants hors de la sphère commune au nom de leur incompatibilité avec le fonctionnement habituel normalisé (RODRIGUES, 2003). L'inclusion peut même exacerber l'exclusion sociale d'enfants issus de populations minoritaires (UNESCO, 2020).

C'est dans ce même mouvement empreint des contradictions de l'inclusif que se développent de nouvelles catégorisations et que se placent de nouveaux professionnels. Alors que les déficiences sensorielles et motrices ne soulèvent plus de problèmes majeurs ni pour les décisions politiques ni pour les aménagements matériels ni pour les enseignants eux-mêmes, c'est tout un secteur de besoins particuliers qui suscite attention, voire crainte et rejet. Parmi ces enfants, ceux qui présentent des difficultés psychiques plus ou moins graves, ceux qui sont confrontés à des difficultés d'apprentissage persistantes (VOLTOLINI, 2014; 2018; SILVA, 2016).

C'est pour ces derniers enfants que le spécialiste est sollicité pour diagnostiquer et traiter: catégorisations en termes de dysfonctionnement des apprentissages (dyslexie, dysorthographie, dyscalculie) ou de perturbations du comportement (troubles de l'attention et hyperactivité), du psychisme (autisme). C'est un retour du spécial non sous sa forme traditionnelle d'institution spéciale mais sous la figure du spécialiste, parfois accompagnée 
http://dx.doi.org/10.5902/1984686X55337

d'un menu psycho pharmaceutique (SILVA, 2016, p.160-161). Au Brésil, se met en place une nouvelle psychopathologie de l'inclusion sous les habits modernistes de l'accueil de tous mais qui traite certains de manière diagnostique pour leur non-conformité (NABUCO, 2010).

Une représentation plus dialectique des relations entre inclusion et exclusion est donc nécessaire. II serait heuristique de renoncer à une vision en tout ou rien, de concevoir l'inclusion non comme un état mais comme un processus susceptible de variations, empreint d'embuches et de paradoxes, non comme un donné mais comme un travail d'affiliation impliquant un ensemble d'acteurs plus ou moins en cohérence. Inclure signifierait alors non plus appliquer rigidement des injonctions technico administratives mais rechercher pragmatiquement les solutions provisoirement acceptables pour accueillir la diversité des enfants dans le milieu scolaire ordinaire, en fonction des conditions locales et des capacités des acteurs de l'éducation.

\section{L'inclusion face à l'intégration}

Un autre pôle de démarcation de l'éducation inclusive se situe dans le cadre de l'évolution de l'éducation spéciale. Celle-ci est historiquement marquée par une culture de la séparation. La mise à l'écart des enfants en situation de handicap a longtemps été considérée comme une mesure nécessaire, justifiée par leurs caractéristiques hors norme scolaire. Or, avec l'éducation inclusive, l'accent est porté sur la diversité et non plus sur des pathologies à dimension médicale. Mais si l'on conçoit bien cette rupture à la fois conceptuelle, organisationnelle et pédagogique par rapport à l'éducation spéciale, peut-on aussi entrevoir une différence par rapport aux mesures d'intégration scolaire qui ont été soutenues dans plusieurs pays et par exemple en France, officiellement, au début des années 80 ?

L'étymologie du mot intégration suggère elle-même plus une normalisation, au sens de l'estompement de toute différence, qu'un changement de paradigme: en latin integrare c'est rendre intégral et non permette l'accès aux droits. Pourtant, certains auteurs ont pu mettre en doute le paradigme inclusif en le taxant d'artifice sémantique, en pointant le risque d'annuler les actions de soutien spécialisé, au nom de la non-discrimination.

Pour répondre à ces critiques, les meilleures clarifications conceptuelles émanent de spécialistes britanniques, sans doute plus attentifs aux terminologies du fait de leur plus grande proximité linguistique avec le vocable de l'inclusion pour désigner des politiques 
http://dx.doi.org/10.5902/1984686X55337

sociales et éducatives. Pour ces auteurs, l'intégration se situe encore dans la continuité de l'éducation spéciale, en se référant seulement à des dispositifs techniques et administratifs pour qu'un enfant handicapé ou un groupe d'enfants handicapés puisse fréquenter, au moins partiellement, un milieu scolaire ordinaire. Elle ne réclame pas de l'école un changement radical dans sa culture et dans son organisation parce que l'attente réside dans l'accommodement de l'enfant aux structures et aux pratiques existantes (BARTON; ARMSTRONG, 2007, p.10).

De plus, les élèves intégrés dans les écoles ordinaires ont le statut de simples visiteurs et non celui de membres à part entière de la communauté scolaire. L'éducation inclusive repose, au contraire, sur le postulat que tous les enfants ont le droit de fréquenter l'école de leur quartier, quelle que soit leur différence. Ce qui implique, pour Barton et Armstrong, une transformation culturelle et éducative de l'école de telle sorte que tous les enfants de la communauté environnante soient accueillis. L'accent est alors porté sur la constitution d'écoles inclusives qui reposent sur la communauté environnante, en termes à la fois de savoirs et de processus d'éducation. Les écoles communes devraient alors reçoivent des appuis pour qu'elles puissent effectivement développer des cultures et des pratiques inclusives et être en mesure de répondre au droit de chaque enfant.

Le renversement de la notion d'adaptation est alors essentiel (THOMAZET, 2008 ; PLAISANCE; BELMONT; VERILLON, 2007). L'intégration exige l'adaptation de l'enfant à un contexte d'accueil et réclame donc de sa part des efforts parfois insurmontables pour se fondre dans un milieu scolaire normalisé. L'éducation inclusive se réfère, à l'inverse, à l'adaptation du milieu scolaire à la diversité enfantine. De manière encore plus prospective, de nombreux auteurs ou des organismes internationaux définissent l'inclusion comme un processus de changement visant à vaincre les barrières aux apprentissages et à la participation de l'ensemble des apprenants (BOOTH et al., 2000; KRON; PLAISANCE, 2012; KRON; SERRANO; AFONSO, 2014). Le point central se concentre alors sur la capacité des systèmes éducatifs à répondre à la diversité. Le professeur inclusif doit luimême être capable d'identifier les barrières à la participation et aux apprentissages, être réflexif, être un penseur critique capable de résoudre des problèmes, en sachant mettre en question la discrimination (DONNELLY; WATKINS, 2011; UNESCO, 2013; KALUBI; GREMION, 2015). 
http://dx.doi.org/10.5902/1984686X55337

\section{Avec Durkheim et au-delà de Durkheim}

J'ai appliqué la notion durkheimienne de théorie pratique à la notion d'inclusion de manière un peu provocatrice pour tenter de lever les nombreuses ambiguïtés des usages actuels en matière d'éducation. Pourtant, les sciences humaines et sociales ont profondément évolué depuis l'époque de Durkheim et elles modifient nos regards sur les pratiques elles-mêmes en les posant comme objets de recherche scientifique. Ces analyses contribuent fortement à débusquer les équivoques, voire les contradictions.

Les travaux d'orientation psychanalytique portant sur les discours de l'inclusion dévoilent le risque de soutenir une injonction universalisante au nom d'une certaine idée du Bien et de la transformer en une chimère qui ignore les singularités, par exemple celle du sujet psychotique (VOLTOLINI, 2014; 2019). Au Brésil, l'inclusion, analysée à partir de discours tenus dans une revue pédagogique représentative d'orientations nationales (Nova Escola), se révèle idéalisée comme un nouvel impératif scolaire parasité par la valorisation de savoirs techno-scientifiques spécialisés et, parallèlement, par la dévalorisation de l'expérience du professeur (SILVA, 2016, p. 56).

Les chercheurs en sciences sociales s'attachent à repérer l'arrière-plan sociétal qui explique et soutient à la fois les orientations inclusives. Si le mot inclusion s'est imposé dans les débats internationaux et aussi nationaux, il a d'abord pris prend sens en fonction de la diffusion du modèle social du handicap contestant sa réduction à une dimension individuelle et médicale. Plus largement, il est ancré dans une vision systémique de la société où les organisations locales, dont l'établissement scolaire, sont au premier plan pour assumer la promotion d'une ouverture à la différence et de l'accès aux droits (EBERSOLD, 2009, p. 77). Serait à l'œuvre une rationalité post-normative qui "situe l'acceptation de la différence et les formes de citoyenneté qui peuvent y être liées dans l'aptitude des individus à bâtir le consensus sur le sens que prend la diversité dans l'établissement" (ibid., p. 77).

D'autres auteurs situent l'inclusion en référence au concept de sociologie politique de référentiel d'action publique, qui rassemble des valeurs, des normes, des modes d'explication causales et qui mobilise des acteurs divers (CHAUVIĖRE, 2000). Ce référentiel global de l'inclusion consacre des perspectives d'action plus sectorielles que se donnent différents types d'acteurs, peut-être dans une manière de contrer les effets délétères de la post modernité. Dans cette perspective, l'éduction inclusive peut être une ressource pour la transformation des pratiques éducatives ouvertes à la diversité, mais à condition d'avoir des 
http://dx.doi.org/10.5902/1984686X55337

supports politiques, juridiques et institutionnels, afin d'agir contre les risques de désocialisation du social (CHAUVIÈRE; PLAISANCE, 2000; CHAUVIÈRE, 2011).

Les recherches de terrain offrent aussi des ressources précieuses pour débusquer les pièges inhérents à l'application concrète de l'objectif d'inclusion solaire. A ce titre, les approches de type ethnographique présentent l'avantage de se situer du côté des acteurs, de recueillir leurs représentations, de les observer dans la vie quotidienne. L'éducation inclusive peut alors être montrée dans ses paradoxes et ses difficultés, soit chez les enseignants, soit chez les élèves. Loin de dénoncer l'éducation inclusive, il importe chez ces auteurs de la situer à différents niveaux de complexité. Par exemple, en France, des élèves désignés comme handicapés mentaux qui fréquentent à la fois une école commune et un dispositif d'aide interne à l'école (dans un va et vient entre ce dispositif et la classe commune) témoignent à l'observateur des tensions qu'il vivent dans les interactions avec les autres et dans les processus d'apprentissage. Ces élèves subissent une véritable épreuve sociale dans leurs expériences, du fait même de leur désignation comme handicapés mentaux avec ce que cela représente comme valeurs négatives et disqualifiantes. L'objectif d'inclusion scolaire se heurte ici à des logiques sociales et au caractère normatif de l'institution scolaire (LANSADE, 2019). Les élèves de ce type ne sont pourtant pas dépourvus ni de capacités réflexives ni de volonté de s'affirmer parmi les autres élèves. Si on leur donne cette possibilité, ils peuvent réclamer leur reconnaissance au sein de l'espace commun, par exemple dans une enquête où ils s'adressent à l'ensemble de l'école dans une lettre ouverte pour dire: on a notre place dans l'école, comme vous (SAINT MARTIN, 2019).

\section{Perspectives d'action: vers l'accessibilité?}

II reste néanmoins à apprécier les rapports entre la démarche scientifique et les perspectives d'action, apparemment opposées dans les textes de Durkheim cités plus haut. Mais ces positions n'excluaient pas chez Durkheim lui-même des engagements socio politiques. Rappelons ici sa fameuse phrase sur la sociologie "qui ne vaudrait pas une heure de peine si elle ne devait avoir qu'un intérêt spéculatif" (DURKHEIM, 1893). Et il se situait clairement du côté des républicains de la Troisième République en faveur de l'école primaire gratuite, laïque et obligatoire.

Parmi les recherches contemporaines en sciences sociales, certains courants revendiquent explicitement un rapport étroit entre analyse et orientation d'action. C'est le 
http://dx.doi.org/10.5902/1984686X55337

cas des travaux issus de l'école dite de Francfort, courant de recherche en Allemagne entre la sociologie et la philosophie, qui se définit comme Théorie critique alliant analyse du social, dénonciation critique et perspective émancipatrice. Ce dernier aspect est posé sans ambiguïtés comme une orientation normative visant à modifier les rapports sociaux, comme chez Axel Honneth (2010) qui table ses travaux et ses propositions sur le concept central de reconnaissance 6 .

Nous ne sommes pas délivrés pour autant de la nécessité absolue de mener des analyses validables scientifiquement pour que, précisément, elles puissent avoir un prolongement justifié vers des propositions d'ordre pratique. Est-ce en dépassant les limites de la notion d'inclusion? On a vu certains avantages de l'utilisation de l'adjectif inclusif, susceptible de développer des analyses plus nuancées, plus dialectiques, pour éviter l'aspect trompeusement homogénéisant de l'inclusion et ses déclinaisons qui renvoient parfois aux mesures partielles de l'ancienne intégration (comme dans les expressions aller en classe d'inclusion, élève d'inclusion, utilisées de manière erronée dans certaines écoles françaises).

Pour aller plus loin à la fois dans l'avancement conceptuel et dans les perspectives d'action, l'opérationnalisation des principes revendiqués par l'ambition inclusive pourrait être rapprochée des facteurs intervenant dans l'accessibilisation des environnements scolaires et sociaux pour dépasser les contradictions de la notion d'inclusion. L'accessibilité pourrait ainsi être mobilisée comme concept unificateur permettant d'appréhender l'aggiornamento de l'institution scolaire qui accompagne l'avènement d'un modèle de société se voulant inclusif (EBERSOLD, 2020). L'analyse des conditions d'accessibilisation des environnements scolaires pourrait ainsi ouvrir une voie plus directement pragmatique pour ouvrir à de nouvelles représentations centrées sur l'accueil de la diversité des individus euxmêmes conçus comme recelant des potentialités parfois insoupçonnées. II faut toutefois penser l'accessibilité dans toute l'ampleur de la notion et l'associer à un impératif, c'est-àdire à une prescription morale commandant au système scolaire et, plus généralement, à la société d'être inclusives (EBERSOLD, 2017). Ce nouvel idéal normatif (EBERSOLD, 2019; 2020) est souvent réduit à la mise en place de dispositifs techniques et matériels qui facilitent l'accès pratique à des lieux (rampes, ascenseurs) ou à des communications (boucles magnétiques pour le son, transcriptions entre le son et l'écrit, interactions par ordinateurs). Elle est bien plus ambitieuse si elle n'est plus seulement une facilitation technique mais une modification radicale de la représentation de l'environnement pour qu'il 
soit une ouverture à l'autre, quel qu'il soit, et à sa participation sociale. Dans le champ éducatif, c'est alors d'une accessibilité pédagogique dont nous avons besoin. Elle implique non plus seulement le postulat de l'éducabilité de chacun mais aussi les compétences renouvelées du professeur dans sa responsabilité de construire un environnement éducatif qui ne marginalise pas certains élèves et, au contraire, promeut les capacités de tous (PLAISANCE, 2013; 2020).

\section{Références bibliographiques}

BARTON, Len; ARMSTRONG, Felicity (orgs). Policy, Experience and Change: crosscultural reflections on inclusive education. Dordrecht: Springer, 2007.

BENOIT, Hervé; PLAISANCE, Éric (orgs). L'éducation inclusive en France et dans le monde. La nouvelle revue de l'adaptation et de la scolarisation. Hors-série. n. 5, 2009.

BOOTH, Tony; AINSCOW, Mel; BLACK-HAWKINS, Kristine; VAUGHAN, Mark; SHAW, L. Index for Inclusion: developing learning and participation in schools. Bristol: Centre of Studies for Inclusive Education, 2000.

BOURDIEU, Pierre; CHAMPAGNE, Patrick. Les exclus de l'intérieur. Actes de la recherche en sciences sociales. n. 91-92, p. 71-74, 1992.

BRASIL. Ministério da Educação. Secretaria de Educação Especial. Política Nacional de Educação Especial na Perspectiva da Educação Inclusiva. Brasília: MEC/SEESP, 2008. Disponível em: http://portal.mec.gov.br/seesp/arquivos/pdf/politica.pdf. Acesso em: 23 set. 2020.

CARVALHO, Rosita Édler. Escola inclusiva: a reorganização do trabalho pedagógico. Porto Alegre: Editora Meditação, 2008.

CASTEL, Robert. La montée des incertitudes. Travail, protections, statut de l'individu. Paris: Seuil, 2009.

CHAUVIÈRE, Michel. Naissance et conséquences d'un nouveau référentiel pour l'action publique. In: BARRAL, Catherine; PATERSON, Florence; STIKER, Henri-Jacques; CHAUVIÈRE, Michel (orgs.). L'institution du handicap : le rôle des associations. Rennes: Presses Universitaires de Rennes, p. 245-249, 2000.

CHAUVIÈRE, Michel. L'intelligence sociale en danger: chemins de résistance et propositions. Paris: La Découverte, 2011.

CHAUVIÈRE, Michel; PLAISANCE, Éric (orgs). L'école face aux handicaps: éducation spéciale ou éducation intégrative? Paris: Presses Universitaires de France, 2000. 
CURY, Carlos Roberto Jamil. Réflexions sur les principes juridiques de l'éducation inclusive au Brésil. Égalité, droit à la différence, équité. Recherche et formation pour les professions de l'éducation. n. 61, p. 41-53, 2009.

DE ANNA, Lucia; PLAISANCE, Éric. (orgs). Inclusive Education and 'Special Pedagogy': the Italian Approach. Alter: Revue européenne de recherche sur le handicap, v. 8, n. 2, p. 65-68 (éditorial du numéro spécial), 2014.

DONNELLY, Verity; WATKINS, Amanda. Former les enseignants à l'inclusion en Europe. La nouvelle revue de l'adaptation et de la scolarisation, n. 55, p. 11-16, 2011.

DURKHEIM Émile. La division du travail social. Paris: Alcan,1893. Réédition: Paris :Presses universitaires de France, 1978.

DURKHEIM, Émile. Nature et méthode de la pédagogie. In: Éducation et sociologie, Chapitre II. Paris: Presses universitaires de France, 1980.

EBERSOLD, Serge. Autour du mot «Inclusion». Recherche et formation, n. 61, p. 71-83, 2009.

EBERSOLD, Serge; PLAISANCE, Éric; ZANDER, Christophe. Ecole inclusive pour les élèves en situation de handicap: accessibilité, réussite scolaire et parcours individuels. Rapport de Conférence de comparaisons internationales. Conseil national d'évaluation du système scolaire-CNESCO: Paris, 2016.

EBERSOLD, Serge. L'École inclusive face à l'impératif d'accessibilité. Éducation et sociétés. n. 40, p.89-103, 2017.

EBERSOLD, Serge. Grammaire de l'accessibilité. Éducation et sociétés. n. 44, p. 29-47, 2019 .

EBERSOLD, Serge. L'accessibilité ou l'école réinventée. London: ISTE / Wiley Editions, 2020.

EUROPEAN AGENCY FOR DEVELOPMENT IN SPECIAL NEEDS EDUCATION. Teacher Education for Inclusion: profile of inclusive teachers. Odense, Denmark: European Agency for Development in Special Needs Education, 2012.

EUROPEAN AGENCY FOR DEVELOPMENT IN SPECIAL NEEDS EDUCATION. Organization of Provision to Support Inclusive Education. Odense, Denmark; Brussels, Belgium: European Agency for Development in Special Needs Education, 2013.

FRANCE. Ministère de l'éducation nationale, de la jeunesse et des sports. Loi n`2013-595 du 8 juillet 2013 d'orientation et de programmation pour la refondation de l'École de la République. Disponível em: https://www.education.gouv.fr/loi-ndeg2013-595-du-8juillet-2013-d-orientation-et-de-programmation-pour-la-refondation-de-l-ecole-5618. Acesso em: 22 Sep. 2020. 
http://dx.doi.org/10.5902/1984686X55337

GARDOU, Charles. La société inclusive, parlons-en! II n'y a pas de vie minuscule. Toulouse: Erès, 2012.

GARDOU, Charles. (Org). Handicap, une encyclopédie des savoirs. Des obscurantismes à de Nouvelles Lumières. Toulouse: Erès, 2014.

HONNETH, Axel. La lutte pour la reconnaissance. Paris: Éditions du Cerf, 2010.

KALUBI; Jean-Claude; GREMION, Lise (orgs). Intégration / inclusion scolaire et nouveaux défis dans la formation des enseignants. Montréal: Éditions Nouvelles, 2015.

KRISTEVA, Julia. Lettre au Président de la République sur les citoyens en situation de handicap, à l'usage de ceux qui le sont et de ceux qui ne le sont pas. Paris:

Fayard, 2003.

KRON, Maria; PLAISANCE, Éric (Orgs). Grandir ensemble: l'éducation inclusive dès la petite enfance. Suresnes: Institut National Supérieur de Formation et de Recherche pour L'éducation des Jeunes Handicapés et les Enseignements Adaptés (INSHEA), 2012.

KRON, Maria; SERRANO, Ana Maria; AFONSO, Joana Lima (Orgs). Crescendo juntos. Passos para a inclusão na educação da infância. Porto: Porto Editora, 2014.

LANSADE, Godefroy. De la difficulté à être reconnus «capables» et «compétents» : des adolescents et jeunes adultes désignés handicapés mentaux en quête d'autonomie. Alter: Revue européenne de recherche sur le handicap. vol.13, n. 1, p. 29-42, 2019.

MANTOAN, Maria Teresa Égler. Inclusão escolar: O que é? Por quê? Como fazer? São Paulo: Moderna, 2003.

MICHAILAKIS, Dimitris; REICH, Wendelin. Dilemmas of Inclusive Education. Alter: Revue européenne de recherche sur le handicap. vol. 3, n. 1, p. 24-44, 2010.

DOI: 10.1016/j.alter.2008.10.001

NABUCO, Maria-Eugenia. Práticas institucionais e inclusão escolar. Cadernos de Pesquisa, v. 40, n. 139, p. 63-74, 2010.

NEVES RODRIGUES, Libéria; RAHME FARID, Monica; FERREIRA JATOBA MERCES da ROCHA, Carla. Política de Educação Especial e os desafios de uma perspectiva inclusiva. Educação e Realidade. vol. 44, n. 1, p. 1 - 21, 2019.

PAUGAM, Serge (org). L’exclusion, l'état des savoirs. Paris: La Découverte, 1996.

PELLETIER, Liliane. Le concept d'inclusion et ses défis. Ressources, n. 22, p.10-29, 2020.

PLAISANCE, Éric. Autrement capables: école, emploi, société - pour l'inclusion des personnes handicapées. Paris: Autrement, 2009. 
PLAISANCE, Éric; BELMONT, Brigitte; VERILLON, Aliette; SCHNEIDER, Cornelia. Intégration ou inclusion? Éléments pour contribuer au débat. La nouvelle revue de l'adaptation et de la scolarisation. n. 37, p. 159-164, 2007.

PLAISANCE, Éric. Ética e inclusão. Cadernos de pesquisa, v. 40, n. 139, p.13-43, 2010.

PLAISANCE Éric. De l'accessibilité physique à l'accessibilité pédagogique: vers un renouvellement des problématiques? La nouvelle revue de l'adaptation et de la scolarisation. n. 63, p. 219-230, 2013.

PLAISANCE, Éric. Os desafios da educação inclusiva e o mal estar na educação, In :VOLTOLINI, Rinaldo (org). Retratos do mal-estar contemporâneo na educação. p. 23-42. São Paulo: Editora Escuta Ltda, 2014.

PLAISANCE, Éric. Da educação especial à educação inclusiva: esclarecendo as palavras para definir as práticas. Educação (Porto Alegre, impresso), v. 38, n. 2, p. 230-238, 2015.

PLAISANCE, Éric. La reconfiguratíon du métier d'enseignant dans la dynamique de l'accessibilité. In: EBERSOLD, Serge. L'accessibilité ou l'école réinventée. London: ISTE/Wiley Editions, 2020.

PRUD'HOMME, Luc; DUCHESNE, Hervé; BONVIN, Patrick; VIENNEAU, Raymond (Orgs.). L'inclusion scolaire: ses fondements, ses acteurs, et ses pratiques. Louvain-laNeuve: De Boeck, 2016.

RAHME, Mônica Maria Farid. Inclusion et internationalisation des droits à l'éducation: les expériences brésilienne, nord-américaine et italienne. La nouvelle revue de l'adaptation et de la scolarisation, n. 64, p. 223-240, 2013.

RAVAUD, Jean -François; STIKER, Henri-Jacques. Les modèles de l'inclusion et de l'exclusion à l'épreuve du handicap $1^{\mathrm{e}}$ partie. Handicap: revue de sciences humaines et sociales. n. 86, p. 1-18, $2000 a$.

RAVAUD, Jean -François; STIKER, Henri-Jacques. Les modèles de l'inclusion et de l'exclusion à l'épreuve du handicap $2^{\mathrm{e}}$ partie. Handicap: revue de sciences humaines et sociales. n. 87, p. 1-17, 2000 b.

RODRIGUES, David (org). Perspectivas sobre a inclusão: de educação à sociedade. Porto: Porto Editora, 2003.

SAINT MARTIN, Claire de. La parole des élèves en situation de handicap: on sait marcher droit mais de travers. Grenoble: Presses universitaires de Grenoble, 2019.

SILVA, Kelly Cristina Brandão da. Educação inclusiva: para todos ou para cada um? Alguns paradoxos (in) convenientes. São Paulo: Editora Escuta, 2016.

THOMAZET, Serge. L'intégration a des limites, pas l'école inclusive. Revue des Sciences de l'Éducation. vol. 34, n. 1, p. 123-139, 2008. 
http://dx.doi.org/10.5902/1984686X55337

TOULLEC-THÉRY, Marie (org). Il/elle va en inclusion. De l'énonciation d'un paradoxe sémantique aux pratiques de classe de l'école. Ressources. n. 22, p. 6-8, 2020. (introduction au numéro spécial de la revue)

TREMBLAY, Philippe. Inclusion scolaire: dispositifs et pratiques pédagogiques. Bruxelles, De Boeck Education, 2012.

ULLRICH, Wladimir Brasil. Política de Educação Especial: sobre ambivalência, tensão e indeterminação. Educação e Realidade. vol. 44, n. 1, p. 1-15, 2019.

UNESCO. Déclaration de Salamanque sur les principes, les politiques et les pratiques en matière d'éducation et de besoins éducatifs spéciaux, juin 1994. Disponível em: http://www.unesco.ch/pdf/salamanca. Acesso em: 20 sep. 2020.

UNESCO. Principes directeurs pour l'inclusion dans l'éducation. Paris: Unesco, 2009.

UNESCO. Promoting Inclusive Teacher Education. Advocacy Guides. Paris: Unesco, 2013.

UNESCO. Déclaration d'Incheon. Education 2030. Paris: Unesco, 2015.

UNESCO. Inclusion and education: all means all. Global Education Monitoring Report. Paris: Unesco, 2020.

VOLTOLINI, Rinaldo (org). Retratos do mal-estar contemporâneo na educação. São Paulo: Editora Escuta Ltda, 2014.

VOLTOLINI, Rinaldo. Interpelações Éticas à Educação Inclusiva. Educação e Realidade. vol. 44, n. 1, p. 1-8, 2019.

WARNOCK, Mary; NORWICH, Brahm; TERZI, Lorella (Orgs.). Special Educational Needs: a new look. London-New York: Continuum International Publishing Group, 2010.

\section{Notas}

${ }^{1}$ Conseil créé en 2003 sous la présidence de Julia Kristeva et Charles Gardou, qui jouait le rôle de groupe de pression sur les pouvoirs publics pour modifier les dispositifs pour les personnes en situation de handicap.

2 En anglais, les notions utilisées dans les textes de Salamanque se réfèrent clairement au vocabulaire de I'inclusion. Ainsi: inclusive orientation, inclusive schooling, etc.

${ }^{3}$ Peuvent être cités la Belgique, la République Tchèque ou certaines régions (Länder) d'Allemagne.

${ }^{4}$ Peuvent surtout être cités les pays scandinaves.

${ }^{5}$ Voir, par exemple, les précisions publiées en anglais par De Anna et Plaisance (2014).

${ }^{6}$ Ce concept de reconnaissance mériterait d'être repris et développé précisément en rapport avec le thème de l'éducation inclusive pour signifier avec plus de force l'appartenance de chacun à l'espace commun. 
http://dx.doi.org/10.5902/1984686X55337

\section{Correspondance}

Éric Plaisance - Avenida Niemeyer 845. São Conrado. Rio de Janeiro. CEP 22450221. Brésil.erplaisance@gmail.com

\section{(c) (7) (8)} 4.0 International (CC BY-NC 4.0) 